\title{
Calidad del cuidado de enfermería y la evaluación del desarrollo psicomotor del niño menor de cinco años en el Centro de Salud "Virgen del Carmen" La Era, Lurigancho Chosica - Lima
}

\author{
Quality of the nursing care and the psychomotor development of the children under five years old in \\ the Health centre "Virgen Del Carmen" La Era, Lurigancho Chosica - Lima
}

Marvic Bautista Melendrez¹, Thalía Espíritu Ramos', Héctor Huamanchoque Chuctaya²

\begin{abstract}
RESUMEN
Objetivo: Determinar la asociación entre la calidad del cuidado de Enfermería y el desarrollo psicomotor de los niños menores de cinco años del Centro de Salud Virgen del Carmen - La Era. Material y métodos: Diseño no experimental, enfoque cuantitativo, alcance descriptivo-correlacional y de corte transversal. El tipo de muestreo fue probabilístico, la muestra estuvo conformada por 281 niños con sus respectivas madres. Se utilizó tres instrumentos: Escala de Calidad del Cuidado de Enfermería adaptada, Guía de Evaluación de 0 a 30 mesesTest Peruano; Guía de Evaluación de 3 y 4 años-Pauta Breve. Resultados: Se encontró que el $89.7 \%$ de las madres refirieron que la calidad del cuidado de Enfermería es alta, $10 \%$ media. Y en la evaluación de desarrollo psicomotor de los niños de 0 a 30 meses, el $97.6 \%$ presentaron desarrollo psicomotor normal, y solo un $2.4 \%$, riesgo de trastorno del desarrollo. De los niños de 3 y 4 años, el $85.2 \%$ presentan desarrollo psicomotor normal. Conclusiones: Se concluye que existe asociación significativa entre calidad del cuidado de Enfermería y la evaluación del desarrollo psicomotor $(\mathrm{p}=, 000)$.
\end{abstract}

Palabras clave: Percepción, calidad, madres, desarrollo psicomotor, niño.

\begin{abstract}
Objetive: The objective was to determine the association between quality of nursing care perceived by mothers and psychomotor development of children under five years old in the "Virgen del Carmen Health Centre- The Era", Lurigancho. Methods: The methodology was non-experimental, quantitative approach, descriptive-correlational reach and cross cut. The type of the sample was probabilistic; the sample was composed by 281 children with their mothers. Tree instruments were used: Adapted Quality Scale of Nursing Care, Evaluation Guide of 0-30 months- Peruvian Test, Evaluation Guide of 3 and 4 years-Brief pattern. Results: The results showed the $89.7 \%$ of mothers perceived a high quality, $10 \%$ medium and $0.4 \%$ lower. Children $0-30$ months $97.6 \%$ present normal psychomotor development, 2.4\% risk to the disorder of the development. About the 3 and 4 years old children, the $14.8 \%$ have a psychomotor development deficit and the $85.2 \%$ normal. Conclusions: In conclusions is significant association between quality of nursing care and evaluation of psychomotor development $(p=.000)$.
\end{abstract}

Keywords: Perception, quality, mothers, psychomotor development, child.

${ }^{I}$ Licenciada en Enfermería, Universidad Peruana Unión, Lima, Perú.

${ }^{2}$ Licenciado en Enfermería, Coordinador de Emergencia Pediátrica, Servicio de Emergencia, Hospital Vitarte. Lima, Perú. 


\section{INTRODUCCIÓN}

Una de las causas de consultas frecuentes en el campo neuropediátrico es el retraso del desarrollo neurológico, por lo que es tarea fundamental el detectar precozmente el retraso (Pucllas, 2003).

Uno de cada diez pacientes adultos o pediátricos, vistos en atención primaria, tiene problemas neurológicos. Es así que la prevalencia de trastornos del desarrollo se ha estimado en $16 \%-18 \%$ de los niños, de los cuales alrededor del $90 \%$ se relacionan a problemas de aprendizaje, lenguaje o retardo mental. Esta cifra aumenta a $22 \%$, si se incluyen problemas del comportamiento (De los Ángeles, 2005).

Se estima que el $10 \%$ de la población de cualquier país está constituido por personas con alguna discapacidad, se entiende por persona con discapacidad a aquella que presenta alguna dificultad permanente física o mental. Uno de los grupos más vulnerables y desprotegidos son los niños, siendo los más afectados por la pobreza, no solo porque su bienestar y calidad de vida dependan de las decisiones de sus padres, del entorno familiar y comunitario, sino también por las condiciones en la que se desenvuelve el niño. El desarrollo integral de la infancia es fundamental para el desarrollo humano y la construcción de capital social, elementos considerados principales y necesarios para romper el ciclo de la pobreza y reducir las brechas de inequidad (OMS, 2011).

Dentro de los lineamientos de política peruana, se considera que la estrategia es priorizar y consolidar las acciones de atención integral con énfasis en la promoción y prevención, disminuyendo los riesgos y daños de los niños; por lo que el rol de la enfermera en el componente Crecimiento y Desarrollo (CRED) está orientado a promover la salud física, mental y emocional del niño, así como el de la madre. Por esta razón es indispensable desarrollar una buena relación con los padres y/o acompañantes del niño, a través de mensajes claros y sencillos, respetando costumbres, brindando un trato amable y cálido, aplicando todos los conocimientos para la atención integral y de calidad (Mellado, 2007).

Es deber, de la enfermera comunitaria, educar a la población especialmente a las madres para que puedan cuidar de sus hijos y evitar cualquier tipo de riesgo o retraso en el menor de cinco años. Sin embargo, la calidad del cuidado que brinda el profesional de Enfermería está cada vez en decadencia, es decir, que hay un gran porcentaje de personas que categorizan el cuidado de Enfermería entre medio y bajo, debido a que el profesional de Enfermería no se abastece por la demanda de pacientes o usuarios que cuida/atiende. En su investigación sobre calidad del cuidado de Enfermería y crecimiento y desarrollo del niño menor de cinco años en el consultorio de CRED, demostró que $83 \%$ de las madres percibieron que la calidad del cuidado de la enfermera es regular y malo, de las cuales sus niños menores de 5 años presentaron desarrollo alterado. (Vílchez \& Gorritti, 2007)

El desarrollo psicomotor del niño requiere de la participación activa de distintos factores, los cuales pueden favorecer o entorpecer el adecuado desenvolvimiento del niño en el medio que lo rodea. Dentro de los factores que pueden influir, en el desarrollo de un niño, podemos definir variables como una buena nutrición, estimulación sensorial adecuada $\mathrm{y}$ oportuna, un sólido vínculo madre-hijo y una buena interrelación enfermera-madre. El Ministerio de la Salud (MINSA, 2010) destaca la importancia del desarrollo en la primera infancia con respecto a la formación de la inteligencia, la personalidad y el componente social. Pues, si los niños y niñas no reciben en esos años formativos la atención y el cuidado que necesitan, las consecuencias serán acumulativas y prolongadas. Estas consideraciones llevaron a realizar la presente investigación, con el objetivo de determinar la asociación entre la calidad del cuidado de Enfermería y el nivel de evaluación del desarrollo psicomotor del niño menor de cinco años, en el Centro de Salud Virgen del Carmen- La Era, Lurigancho, Lima Perú.

\section{MATERIALES Y MÉTODOS}

El estudio se realizó en el Centro de Salud "Virgen del Carmen"-La Era, Lurigancho Chosica, durante el año 2014. La investigación fue de diseño no experimental, de enfoque cuantitativo, de alcance descriptivo correlacional y de corte transversal. El tipo de muestreo fue probabilístico, considerándose una muestra de 281 niños con sus respectivas madres, captadas en el servicio de CRED.

Los instrumentos usados fueron tres: La Escala de Calidad del Cuidado de Enfermería constituido por tres dimensiones específicas: técnico-científico, humana y entorno-confort; fue previamente validado por juicio de expertos y fue aplicado a las madres. Tuvo una confiabilidad de Alfa de Cronbach de 0.870 . Para evaluar el desarrollo psicomotor de los niños menores de 5 años, se usó la Guía de Evaluación de 0 a 30 meses-Test Peruano y la Guía de Evaluación de 3 y 4 años-Pauta Breve, siendo el uso de estos normados por el Ministerio de Salud del estado peruano. 
La recolección de datos fue previa autorización del director del Centro de Salud y el consentimiento de las madres. La información fue procesada con el paquete estadístico SPSS 20.0 y la prueba estadística usada fue el Chi-cuadrado.

\section{RESULTADOS}

Tabla 1

Calidad del cuidado de enfermería según la percepción de las madres de los niños menores de 5 años en el Centro de Salud "Virgen del Carmen" - La Era,2014.

\begin{tabular}{lcc}
\hline Nivel de calidad & N & $\%$ \\
\hline Baja calidad & 1 & 0.4 \\
Calidad media & 28 & 10 \\
Alta calidad & 252 & 89.7 \\
Total & 281 & 100 \\
\hline
\end{tabular}

En la tabla 1 se observa que existe un nivel alto de de calidad media $10 \%$ y solo $0.4 \%$ indica que existe calidad del cuidado de enfermería (89.7\%), seguido un nivel de calidad baja.

Tabla 2

Evaluación del Desarrollo Psicomotor del niño menor de 5 años en el Centro de Salud "Virgen del Carmen" - La Era, 2014.

\begin{tabular}{lcc}
\hline Evaluación del desarrollo & $\mathrm{N}$ & $\%$ \\
\cline { 2 - 3 } Psicomotor & & \\
\hline Trastorno del desarrollo & 0 & 0 \\
Déficit/Riesgo del desarrollo & 10 & 3.6 \\
Normal & 271 & 96.4 \\
Total & 281 & 100.0 \\
\hline
\end{tabular}

En la tabla 2 se muestra que del $100 \%$, el $96.4 \%$ de el $3.6 \%$ déficit/riesgo del desarrollo, en tanto que no los niños evidencian un desarrollo psicomotor normal, hay niños que muestren trastorno del desarrollo.

Tabla 3

Calidad del cuidado de enfermería según la percepción de las madres y el nivel de evaluación del desarrollo psicomotor del niño de 0 a 30 meses en el Centro de Salud Virgen del Carmen - La Era, 2014.

\begin{tabular}{lllllll}
\hline \multirow{2}{*}{ Nivel de calidad } & \multicolumn{2}{c}{$\begin{array}{l}\text { Resgo para el } \\
\text { trastorno }\end{array}$} & \multicolumn{2}{c}{ Desarrollo normal } & \multicolumn{2}{c}{ Total } \\
\cline { 2 - 8 } & $\mathrm{N}$ & $\%$ & $\mathrm{~N}$ & $\%$ & $\mathrm{~N}$ & $\%$ \\
\hline Baja calidad & 0 & 0 & 1 & 0.4 & 1 & 0.4 \\
Calidad media & 0 & 0 & 25 & 10 & 25 & 10 \\
Alta calidad & 6 & 2.1 & 222 & 87.5 & 228 & 89.7 \\
Total & 6 & 2.1 & 248 & 97.9 & 254 & 100 \\
\hline
\end{tabular}

Chi cuadrado $(p=.003)$.

En la tabla 3 , se muestra que el $87.2 \%$ presenta un nivel de calidad del cuidado de enfermería alta $\mathrm{y}$, a la vez, los niños evidencian desarrollo psicomotor normal, entre tanto, solo el $0.4 \%$ indica un nivel de calidad baja, y los niños presentan un déficit/riesgo del desarrollo en el Centro de Salud Virgen del Carmen - La Era. 
Calidad del cuidado de enfermería y la evaluación del desarrollo psicomotor del niño menor de cinco años en el Centro de Salud "Virgen del Carmen" La Era, Lurigancho Chosica - Lima

Tabla 4

Calidad del cuidado de enfermería según la percepción de las madres y el nivel de evaluación del desarrollo psicomotor del niño de 3 y 4 años en el Centro de Salud Virgen del Carmen - La Era, 2014.

\begin{tabular}{lllllll}
\hline \multirow{2}{*}{$\begin{array}{l}\text { Nivel de } \\
\text { Calidad }\end{array}$} & \multicolumn{2}{c}{ Déficit } & \multicolumn{2}{c}{ Normal } & \multicolumn{2}{c}{ Total } \\
& N & $\%$ & N & $\%$ & N & $\%$ \\
\hline Baja calidad & 0 & 0 & 0 & 0 & 0 & 0.4 \\
Calidad media & 1 & 0.4 & 3 & 9.6 & 4 & 10 \\
Alta calidad & 2 & 2.5 & 21 & 87.2 & 23 & 89.7 \\
Total & 3 & 2.9 & 24 & 96.8 & 27 & 100 \\
\hline
\end{tabular}

Chi cuadrado $(p=.000)$

De la población en estudio, en cuanto al desarrollo psicomotor, se observa que el $87.2 \%$ indica un nivel de calidad del cuidado de Enfermería como alta y los niños presentaron un desarrollo psicomotor normal, por otro lado, el $0.4 \%$ indica el nivel de calidad como baja y los niños presentaron un déficit en cuanto al desarrollo, en el Centro de Salud Virgen del Carmen - La Era, tal como se observa en la tabla 4.

\section{Tabla 5}

Calidad de cuidado de enfermería según la percepción de las madres y el nivel de evaluación del desarrollo psicomotor del niño menor de 5 años en el Centro de Salud Virgen del Carmen - La Era, 2014.

\begin{tabular}{|c|c|c|c|c|c|c|c|c|}
\hline \multirow{3}{*}{ Nivel de calidad } & \multicolumn{8}{|c|}{ Evaluación del Desarrollo Psicomotor } \\
\hline & \multicolumn{2}{|c|}{$\begin{array}{l}\text { Trastorno del } \\
\text { Desarrollo }\end{array}$} & \multicolumn{2}{|c|}{$\begin{array}{l}\text { Déficit/Riesgo del } \\
\text { Desarrollo }\end{array}$} & \multicolumn{2}{|c|}{ Normal } & \multicolumn{2}{|c|}{ Total } \\
\hline & $\mathrm{N}$ & $\%$ & $\mathrm{~N}$ & $\%$ & $\mathrm{~N}$ & $\%$ & $\mathrm{~N}$ & $\%$ \\
\hline Baja calidad & 0 & 0 & 1 & 0.4 & 0 & 0 & 1 & 0.4 \\
\hline Calidad media & 0 & 0 & 1 & 0.4 & 27 & 9.6 & 28 & 10 \\
\hline Alta calidad & 0 & 0 & 8 & 2.8 & 244 & 86.8 & 252 & 89.7 \\
\hline Total & 0 & 0 & 10 & 3.6 & 271 & 96.4 & 281 & 100 \\
\hline
\end{tabular}

De la población, de 281 madres y sus niños menores de cinco años, el $86.8 \%$ indica un nivel de calidad del cuidado de Enfermería alta y, a su vez, los niños presentaron desarrollo psicomotor normal, entre tanto, solo el $0.4 \%$ indica un nivel de calidad como baja y los niños presentaron un déficit/riesgo del desarrollo en el Centro de Salud Virgen del Carmen - La Era, tal como se observa en la tabla 5.

\section{Tabla 6}

Relación entre la calidad del cuidado de enfermería percibido por las madres y el desarrollo psicomotor del niño menor de cinco años.

\begin{tabular}{lccc}
\hline & Valor & gl & p \\
\hline Chi-cuadrado de Pearson & $27.208^{\mathrm{a}}$ & 2 & .000 \\
\hline N de casos válidos & 281 & & \\
\hline
\end{tabular}

Mediante la prueba estadística, como se observa en la tabla 6 , se percibe un nivel de significancia alta $(\mathrm{p}=0,00)$, el cual es menor al nivel de significancia establecido $(\mathrm{p}<0,05)$, por tanto, existe asociación entre el nivel de calidad del cuidado de Enfermería y la evaluación del desarrollo psicomotor del niño menor de cinco años. 


\section{DISCUSIÓN}

Los profesionales de Enfermería, quienes trabajan en el programa de crecimiento y desarrollo, deben disponer de talento humano y estar capacitados en el tema, con el fin de brindar atención humanizada y de calidad, que garantice intervenciones eficientes, oportunas y adecuadas. La enfermera es una educadora por excelencia, ya que siempre se dirige a los cuidadores familiares, a la comunidad y también al niño para lograr un adecuado crecimiento y desarrollo infantil. Contribuye en su desarrollo integral, enriquece su formación y fomenta su realización dentro de la sociedad del futuro (Vílchez \& Gorritti, 2007).

Guevara (2013) menciona que la calidad de atención o cuidado de Enfermería es entender que el usuario es quien define la calidad que brinda la enfermera, además, es esforzarse por complacer al usuario y no conformarse únicamente con resolver sus problemas inmediatamente.

Es así que el presente estudio indagó sobre la calidad de cuidado de Enfermería en el Centro de Salud Virgen del Carmen, La Era, obteniendo como resultado que el $89.7 \%$ indica un nivel de calidad de cuidado alto, este resultado es porque la realización de las actividades son de gran importancia en el control de crecimiento y desarrollo de los niños, pues no solo direccionan muchas de las actividades que la enfermera programa para la atención integral del niño, sino también benefician el proceso de atención al establecer una relación de respeto, confianza y empatía con los padres o cuidadores, además que permite identificar claramente las necesidades de salud del niño y, por tanto, brindar una adecuada atención y consejería, promoviendo una colaboración y participación activa de los padres o cuidadores durante la atención del niño, por tanto, constituyen el primer paso para brindar una atención adecuada en el niño (Martínez, 2011).

Cabe mencionar que los indicadores más resaltantes, en cuanto al nivel de calidad del cuidado del profesional de Enfermería, son: confidencialidad al momento de la consulta (55.5\%), respeto por el niño al ser llamando por su nombre $(60.5 \%)$, demuestra cordialidad y amabilidad en todo momento, respeta el orden de llegada para la atención (36.3\%), muestra interés por el estado de salud del niño $(39.1 \%)$, respeta las creencias y costumbres (49.1\%), siempre está dispuesta a responder las dudas que la madre tiene (42.7\%) y, de ser necesario, acude a los hogares en caso de inasistencia a alguna cita (37\%), asimismo, la enfermera tiene conocimientos suficientes respecto a la evaluación $(63.7 \%)$ o procedimientos usando todo los materiales disponibles $(35.2 \%)$, el ambiente de consulta es propicio para la evaluación del niño, teniendo orden y limpieza $(26 \%)$, presentando iluminación $(31.7 \%)$ y una decoración llamativa para los niños (35.9\%) (Ver tabla 13, anexo 6), estos aspectos técnico-científico, humano y de entornoconfort realizados por el profesional de Enfermería conllevan a que el usuario tengan una percepción de calidad de atención alta. Estos resultados corroboran lo afirmado por el Colegio de Enfermeros del Perú (2008), en el sentido que la calidad de atención del personal de Enfermería debe ser oportuna, personalizada, humanizada, continua y eficiente, de acuerdo a estándares definidos para una práctica profesional competente y responsable. Dicho de otra forma, el cuidado de Enfermería debe prestar ayuda eficiente y efectiva a la persona, a la familia y a la comunidad, fundamentada en los valores y estándares técnicos, científicos, sociales, humanos y éticos.

Otros estudios desarrollados en centros de atención de primer nivel, respecto a la percepción del usuario sobre la calidad de atención de Enfermería, evidencia que el $32 \%$ percibe un nivel alto de satisfacción (Mellado, 2007), entre tanto, Guevara (2013) encontró que el 31\% percibe como favorable la atención de la enfermera. Estos resultados si bien están relacionados con el estudio, llama la atención porque la percepción de la calidad del cuidado que brinda la enfermera al usuario es menor del 50\%, consideramos que esta diferencia se debe a que no mantienen relación de confianza, respeto, empatía y participación activa con los padres durante la evaluación del niño (Martínez, 2011). Mientras que en nuestra población de estudio, la enfermera mantiene una relación de respeto, confianza, empatía y participación activa con los padres.

Una de las funciones prioritarias del profesional de Enfermería comunitaria es evaluar el desarrollo psicomotor de los niños, con la finalidad de diagnosticar, de manera precoz, la existencia de algún riesgo o retraso.

En tal sentido, los resultados de la investigación muestran que la mayoría de los niños menores de cinco años presenta un desarrollo psicomotor normal, en $96.4 \%$. Del grupo etario de 0 a 30 meses; evaluado con el Test Peruano, el $97.6 \%$ se encuentra con desarrollo normal, entre tanto que el grupo de $3 \mathrm{y}$ 4 años, evaluado con la Pauta Breve, evidenció que el $85.2 \%$ presenta desarrollo normal. Esto es porque la realización de las actividades del profesional de Enfermería, en el control de crecimiento y desarrollo de los niños, identifica claramente las necesidades de salud del niño y, por tanto, brinda una adecuada atención y consejería, promoviendo una colaboración y participación activa de los padres o cuidadores 
durante la atención del niño, por tanto, constituyen el primer paso para brindar una atención adecuada en el niño (Martínez, 2011).

Por otro lado, Pucllas (2003) refiere que el niño necesita escuchar palabras de aliento y de cariño, recibir caricias y sonrisas, que se le escuche y lo estimulen a responder con sonidos y movimientos, desde los primeros meses de vida. Asimismo, la atención que recibe de sus padres para lograr un desarrollo físico y mental normal es indispensable, recordando que el niño necesita gozar la libertad para explorar y jugar, el juego es una actividad esencial del proceso de crecimiento, ya que favorece las habilidades mentales, sociales y físicas, dentro de las que se destacan la capacidad de hablar y caminar. La creatividad de un niño requiere de estímulos para que se desarrolle al máximo, donde busque soluciones de los problemas más frecuentes, para poner en práctica sus ideas y decisiones, entre tanto, para que los niños tengan un buen crecimiento y desarrollo se necesita de responsabilidad de los padres y de los educadores iniciales, quienes deben tomar en cuenta que estos dos procesos se encuentran ligados y que son parte de las etapas de evolución del ser humano y que se facilitará su proceso a base del conocimiento, de la experiencia y del cariño.

Tique y Ramos (2012), en su trabajo de investigación sobre crecimiento y desarrollo en niños de 8 a 24 meses, utilizando en EEDP, obtuvieron que el $72 \%$ presenta un desarrollo normal. En nuestro estudio se obtuvo un porcentaje alto en cuanto al desarrollo psicomotor normal del niño, debido a que la enfermera educa a la madre y, por ende, ella aplica lo aprendido, mientras que el estudio presentado se difiere con los resultados, ya que el ambiente y la estimulación que la madre prodiga al niño no es la adecuada.

Otra investigación hecha por Cosme (2007), donde utiliza la Escala del Desarrollo Abreviada, evidencia que el $71.44 \%$ menores de 5 años presenta desarrollo psicomotor medio en la comunidad suburbana de México. Estos resultados difieren con nuestros resultados, esta diferencia se debe a factores intervinientes.

En una investigación, Martínez y Urdangarin (2005) aplicaron tres herramientas diferentes para medir el desarrollo psicomotor del niño tales como Escala de Evaluación del Desarrollo Psicomotor, de Soledad Rodríguez (EEDP); La Escala del Desarrollo Psicomotor de la Primera Infancia de Brunet y Lézine (EEDPI) y el Diagnóstico Funcional según el Método de Múnich (DFDM), se encontró que el desarrollo normal es del 39\% según el EEDP, asimismo, el 78\% según el EDPPI y, por último, el 4\% según el DFDM en una muestra de 55 niños menores de un año, en la Región Metropolitana de Chile. Mientras tanto, en el estudio, aplicando el Test Peruano y la Pauta Breve, se demostró que los niños evaluados presentan desarrollo psicomotor normal en $97.6 \%$.

En cuanto a la asociación entre las variables, se dividió en dos grupos: primero, el nivel de calidad del cuidado de Enfermería y segundo, el desarrollo psicomotor del niño de 0 a 30 meses, donde se demostró con la prueba estadística que existe relación con una significancia alta $(\mathrm{p}=.003)$, seguidamente, la prueba estadística entre calidad del cuidado de Enfermería y el desarrollo psicomotor del niño de 3 y 4 años, se demostró que existe una relación con significancia alta $(\mathrm{p}=.000)$, a nivel general se encontró que existe asociación entre la calidad del cuidado de Enfermería y el desarrollo psicomotor del niño menor de cinco años con una significancia alta $(\mathrm{p}=.000)$. En un estudio similar, realizado por Vílchez y Gorritti (2007), sobre calidad del cuidado de Enfermería percibido por el cuidador familiar y el crecimiento y desarrollo del niño menor de cinco años, demostró también que existe asociación $(p=.004)$ entre las variables. Por tanto, queda demostrado que la alta competencia de la enfermera, el compromiso profesional con la disciplina, el trato humanizado y la ética profesional, permite brindar un cuidado holístico que satisface las expectativas del usuario, lo cual fortalece la interacción enfermera-madre.

En el quehacer de la enfermera, uno de los aspectos más importantes es enseñar e informar, a la madre, la importancia de la estimulación del niño y las actividades a realizar con el niño en sus diferentes áreas del desarrollo, estos cuidados se brindan en la etapa de vida del niño en la prevención de riesgos que afectan el desarrollo normal del niño y promover la salud y vigilancia del desarrollo (Martínez, 2011).

Los resultados obtenidos permiten deducir que el ambiente donde se desenvuelven es el adecuado, es decir, las madres cumplen con las indicaciones del profesional de Enfermería dadas en el consultorio CRED, para así estimular a sus niños a través de sus diferentes áreas en el adecuado crecimiento y desarrollo. Es evidente que existe una fluida interacción enfermera-madre, lo cual conlleva a realizar acciones conjuntas en favor del niño o niña. Al respecto, Castillo (2013) refiere que el desarrollo psicomotor es el resultado de la interacción entre la herencia que aporta la potencialidad del niño y el ambiente que influye en cuánto y cómo se expresará ese potencial, de modo que para lograr un desarrollo óptimo, el entorno debe cubrir las necesidades físicas y psicológicas del niño, de acuerdo a sus características individuales, edad 
y etapa del desarrollo. Quispe (2012) agrega que la calidad de vida del niño depende de las decisiones de sus padres, del entorno familiar y comunitario. Lo que se puede decir es que son importantes las condiciones en la que se desenvuelve el niño. Es deber del profesional de enfermería comunitaria velar por el bienestar del niño/niña realizando la estimulación temprana, educando a las madres y a la comunidad en general. Además, su influencia en la comunidad y las visitas domiciliarias que realiza puede lograr un cambio en la actitud de la madre a favor del niño/niña. Por ende, la enfermera (o) juega un rol protagónico en el crecimiento y desarrollo del niño menor de cinco años.

Álvarez (2007) afirma que el propósito del enfermero es brindar el mejor nivel del cuidado, no menos que excelente, en cuanto a calidad técnica, humana y ética; implica un profundo compromiso profesional con la disciplina, todo ello enmarcado por inflexible responsabilidad, depuradas relaciones humanas y continuada dedicación personal.

Además, la teoría del cuidado de Swanson afirma que los cuidados se dan como un conjunto de procesos secuenciales, creados por la propia actitud filosófica de la enfermera (mantener creencias), la comprensión (conocimiento), los mensajes verbales y no verbales transmitido al cliente (estar con), las acciones terapéuticas (hacer por y posibilitar) y las consecuencias de los cuidados (desenlace deseado por el cliente). Es decir, la base fundamental de Enfermería es brindar un cuidado de calidad, velando por el bienestar del usuario y fomentando el lazo de enfermera-usuario-entorno, con la única finalidad de promover, prevenir y efectivizar su recuperación de su estado de salud de manera integral (Marnner \& Raite, 2007).

Llegando a la conclusión que el nivel de calidad de cuidado de Enfermería, en su mayoría, es alta, del Centro de Salud Virgen del Carmen. Además, la mayoría de los niños de 0 a 30 meses, del Centro de Salud "Virgen del Carmen - La Era", presentan desarrollo psicomotor normal, de acuerdo al Test
Peruano del Desarrollo. Por otro lado, mediante el Test Abreviado, la mayoría de los niños de 3 a 4 años de edad, del Centro de Salud Virgen del Carmen- La Era, tiene desarrollo psicomotor normal.

Existe asociación estadísticamente significativa $(p=003)$ entre la calidad del cuidado de Enfermería y el desarrollo psicomotor de los niños de 0 a 30 meses del Centro de Salud Virgen del Carmen - La Era.

Existe asociación estadísticamente significativa $(p=.000)$ entre la calidad del cuidado de Enfermería y el desarrollo psicomotor de los niños de 3 y 4 años del Centro de Salud Virgen del Carmen - La Era.

Finalmente, en cuanto a las variables de estudio de calidad de atención de Enfermería, percibido por las madres y el nivel de desarrollo psicomotor del niño menor de cinco años, existe asociación estadísticamente significativa $(\mathrm{p}=.000)$. Por tanto, es evidente que una fluida interacción enfermera-madre debe continuar fortaleciéndose.

\section{Declaración de financiamiento y de conflicto de} intereses:

El estudio fue financiado por los autores, quienes declaran no tener algún tipo de conflicto de interés en la investigación realizada.

\section{Correspondencia:}

\section{Thalia Espíritu Ramos}

Universidad Peruana Unión. Carretera Central Km 19.5 Ñaña. Lima. Perú.

e-mail: thaliaespiritu@gmail.com 


\section{REFERENCIAS BIBLIOGRÁFICAS}

Álvarez L., Barrera M. \& Madrigal C. (2007). Calidad de la atención en enfermería. Colombia: CES. Recuperado de:http://bdigital.ces.edu.co:8080/dspace/ bitstream/123456789/26/1/Calidad_atencion_en_ enfermeria.pdf

Carruitero B. (2008). Normas de gestión de la calidad del cuidado enfermero-Colegio de Enfermeros. Recuperado de: http://www.cep.org.pe/beta/download/ NG_CDCE.pdf

Guevara S. (2013). Percepción de las madres acerca de la calidad de atención que brinda la enfermera en el consultorio CRED de la Red de EsSalud "San José" (Tesis de Licenciatura) Universidad Nacional de San Marcos, Lima, Perú.

Martínez C. \& Urdangain D. (2005). Evaluación del desarrollo psicomotor en niños institucionalizados menores de 1 año mediante tres herramientas distintas de evaluación (Tesis de licenciatura, Universidad de Chile). Recuperado de: http://www.tesis.uchile.cl/tesis/ uchile/2005/martinez_c/sources/martinez_c.pdf.

Mellado, C. (2007). Satisfacción de la madre de la atención brindada por la enfermera en el componente CRED del C.M.I. Daniel Alcides Carrión. (Tesis de Licenciatura) Universidad Nacional Mayor de San Marcos, Lima, Perú.

Ministerio de Salud (2010). Monitoreo del crecimiento y desarrollo de las niñas y niños. Recuperado de: http:// www.minsa.gob.pe/portada/prensa/notas_auxiliar. asp? nota $=9207$

Organización Mundial de Salud (2011). Informe mundial sobre la discapacidad. Recuperado de: http://www. who.int/disabilities/world_report/2011/summary_ es.pdf
Pucllas, M. \& Zegarra, G. (2003). Prevalencia de déficit en el desarrollo psicomotor de niños de 2 a 5 años de edad, que asisten a los consultorios de crecimiento y desarrollo del Hospital Docente Materno Infantil San Bartolomé y Hospital Nacional Arzobispo Loayza. (Tesis de licenciatura) Universidad Peruana Unión, Lima, Perú.

Quispe, H. (2012). Nivel de conocimiento y actitud de los padres sobre la estimulación temprana en relación al desarrollo psicomotor del niño de 4 a 5 años de la I. E. Jorge Chávez, Tacna. (Tesis de licenciatura, Universidad Nacional Jorge Basache Grohmann). Recuperado de: http://tesis.unjbg.edu.pe:8080/ bitstream/handle/unjbg/130/24_Quispe_Gutierrez_ HD_FACS_Enfermeria_2012.pdf? sequence $=1$

Tique J. \& Ramos M. (2012). Factores que afectan el Desarrollo Psicomotor de 8 a 24 meses del Jardin Social Perlitas del Otun de Pereira, Colombia. (Tesis de licenciatura en Ciencias del deporte y la Recreación, Universidad Tecnológica, Pereira, Colombia) Recuperado de: http://repositorio.utp.edu.co/dspace/ bitstream/11059/3113/1/37286132T595.pdf

Vílchez, M. \& Gorritti C. (2007). Calidad del cuidado de enfermería percibido por el cuidador familiar y crecimiento y desarrollo del niño menor de cinco años en el consultorio de CRED del P.S. Santa AnaChimbote. (Tesis para obtener la licenciatura) 2(1) 6977. Recuperado de: http://increscendo.uladech.edu.pe/ es/ediciones/ediciones-anuales/2011/vol-2-n-1-enerojunio/item/168-calidad-del-cuidado-de-enfermeriapercibido-por-el-cuidador-familiar-y-crecimientoy-desarrollo-del-ni\%C3\%B1o-menor-de-cinco$\mathrm{a} \% \mathrm{C} 3 \% \mathrm{~B} 1 \mathrm{os}$

Recibido: 19/01/2015 Aceptado: 20/05/2015 